\title{
评述
}

\section{热力学体系的物理学特性与酶学机制的热力学 原理}

\author{
赵钦-*
}

中国康复研究中心医学研究所, 北京 100068

*联系人, E-mail: qinyizhao@gmail.com; Qinyi_zhao@163.com

收稿日期：2017-12-20; 接受日期：2018-03-28; 网络版发表日期：2018-06-13

摘要 本文总结讨论了复杂热力学体系的特点与原理. 在此基础上, 修改了阿累尼乌斯公式, 修改后酶学反应速 率公式包含蛋白质构象变化的热力学参数. 因而可以在理论上分析与确立蛋白质构象变化与酶活性之间的关系. 在本理论体系中，酶的调节与酶的作用机制是蛋白质运动的不同体现，二者的基本原理是一致的. 可以证明，酶 催化过程中活化能的降低来源于酶与底物的结合能. 理论表明基因互作现象是生物信息的热力学整合过程. 综上 所述, 蛋白质热力学是分析任何生物功能与过程的基础与原理.

关键词 蛋白质, 酶, 调节, 机制, 体系

传统热力学是在研究理想气体的物理学行为中建 立起来的. 在理论上, 它们甚至都不能完整, 确切描述 实际气体的各种物理学特征. 因此, 是否能将此理论应 用到复杂热力学体系，特别是生物学体系中是有疑问 的 ${ }^{[1]}$. 但是, 科学的发展证明这些热力学原则能成功处 理很多复杂热力学体系问题, 这些疑问就逐渐消失了. 以至于现在很多人并不考虑这些热力学原则的应用范 围, 仅简单地理解为科学的绝对真理. 科学家的任务就 被人为地简化成为如何灵活应用这些原理到不同领 域, 而它的正确性与应用范围也就无人提及了.

在研究蛋白质酶的热力学问题时发现热力学原则 在处理复杂热力学体系时必须修改，因此本文系统研 究了体系的热力学原理, 在此基础上提出了热力学体 系的配分函数概念，进而推导出酶学速率方程，这些
方程不但能有效地处理酶学反应速率问题，更为重要 的是解决了一系列生物物理学与生物化学的重大问 题: 如酶活性与蛋白质运动之间的关系, 酶的调节机制 与酶活性的基础热力学原理(酶高效催化的原理), 生 物信息的热力学整合原理等 ${ }^{[2 \sim 11}$. 本文试图在统一的 科学基础上阐述这些问题，并对一些历史性科学观点 做出评价.

\section{1 热力学体系的科学定义与热力学原理}

虽然体系是一个很古老的概念，但过去没有体系 的科学定义, 只有哲学理解方式. 在研究蛋白质折叠 时，提出了体系的一个科学定义. 这种定义可以支持 体系科学逻辑的建立及体系各种性质的分析 ${ }^{[12]}$. 蛋白

引用格式: 赵钦一. 热力学体系的物理学特性与酶学机制的热力学原理. 中国科学: 生命科学, 2018, 48: 650-661

Zhao Q Y. Physical characteristics of complex thermal system and working mechanism of enzymes (in Chinese). Sci Sin Vitae, 2018, 48: 650-661, doi: $10.1360 / \mathrm{N} 052017-00298$ 
质研究中发现一种逻辑循环结构, 蛋白质构象调节蛋 白质的运动, 蛋白质运动的耦合又决定蛋白质构象.

蛋白质构象<smiles>[Y][V]</smiles>

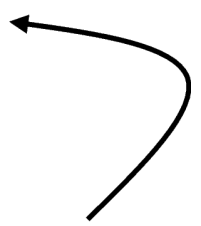

蛋白质运动 $\longrightarrow$ 偶联运动

在逻辑上规定一个含有下列内部结构的物质集合 体为体系:

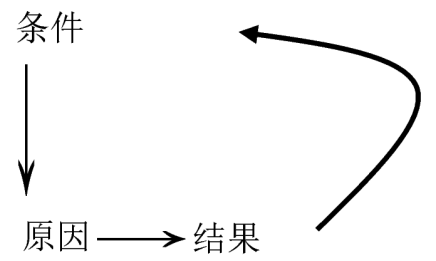

显然，蛋白质内部的热力学体系是这种定义的特 例. 限于篇幅与题材, 本文不考虑体系的逻辑学问题, 仅限于热力学特性的分析. 但需要表明的是, 在科学逻 辑上，体系的这种定义与自然界秩序起源的问题完全 兼容. 蛋白质内部含有规定其结构、形状、体积及其 他性质的一切生物学或物理学信息. 而传统热力学集 合体(气体)的体积不是由本身性质决定的.

在这种定义中, 蛋白质热力学体系代表蛋白质内 部一个热力学运动状态. 它代表蛋白质氨基酸残基的 偶联运动, 蛋白质构象影响蛋白质内部热力学运动, 因此它与一个具体的蛋白质构象相联系, 在理论上可 以用一个具体的物理量(如酶学 $K_{\mathrm{m}}$ )或生物量(酶活性 等)表征这个体系.

在这种定义中，构成体系的各种成分是不能独立 的，体系的性质不能还原成其组成部分的特性．这是 体系的根本特性.

对一个简单体系(或物质集合体):

$$
\begin{gathered}
E_{\mathrm{l}}=\text { constant } \\
E_{\mathrm{W}}=E_{1}+E_{2}+E_{3}+\ldots \\
N_{p}=\sum_{i} e \frac{-E_{i}}{K T},
\end{gathered}
$$

$E$ 代表能量. $E_{\mathrm{I}}$ 代表I组分的能量. $E_{\mathrm{w}}$ 代表体系的能量. $N_{\mathrm{p}}$ 代表组分在某一能级上的概率.

对复杂性热力学体系

$$
\begin{aligned}
& \Delta G= \Delta G_{0}-F(s) \\
& \Delta G= \Delta G_{0}-b\left(s-S_{0}\right) \quad \text { (在线性修订范围) } \\
& E_{\mathrm{W}} \leq E_{1}+E_{2}+E_{3}+\ldots \\
& \quad N_{p s}=\sum_{i} e^{\frac{-\left[\Delta G_{i}^{0}-F_{i}(s)\right]}{K T},}
\end{aligned}
$$

$\Delta G$ 代表体系的自由能. $F$ 代表函数. $b$ 代表修订常数. $s$ 代表某一环境因子的强度. $E \mathrm{w}$ 代表总能量. $N_{\mathrm{ps}}$ 代表体 系在某一状态的概率.

在复杂热力学体系中, 各组分的运动是相互影响 或相互偶联的, 因此其总能量不等于其组成部分的 总和.

在蛋白质科学中, 由于研究方法及研究领域的不 同，不同的科学家用不同的术语描述蛋白质的热力学 体系及特性, 其中有蛋白质热力学结构理论 (potherse) ${ }^{[13]}$ 、蛋白质landscape理论(ensemble of protein conformation) ${ }^{[14]}$ 、构象状态(conformational state, 生物物理学家术语 $)^{[15]}$ 、 Englander蛋白质折叠理论 (foldon) ${ }^{[16]}$ 和蛋白质构象(生物化学家术语). 根据上述 公式, 在理论上可以推导出蛋白质体系不同热力学状 态的分布曲线 ${ }^{[2-4]}$. 在实际中, 可以根据热力学状态的 分布分析其特性及变化机制.

与传统理论(质点体系)不同, 热力学体系的状态 与环境息息相关, 外界因素对它的影响可以由热力学 体系状态的分布曲线来分析. 图1代表传统的热力学 分布与体系状态的热力学分布的比较.

从图 $1 \mathrm{~A}$ 中可以看出, 热力学状态的几率可以在很 小的温度范围内发生根本性变化, 一个状态可以 $100 \%$ 转变成另一种状态. 而电子在高能级中的概率分布变 化发生在大范围内, 而且转变不能达到 $100 \%$, 理论最 高为 $50 \%$. 从理论上考虑, 蛋白质热力学状态的改变仅 牵连很小的能量变化, 一个 $\mathrm{kB}$ 能量 (在室温时约 $0.5 \mathrm{~kJ} / \mathrm{mol}$ ) 就可以引起状态分布的巨大变化, 其能级 的能量是随环境变化而变化的, 和环境因素有关. 而 在传统热力学分布中, 分子量子能级间的能量差别是 恒定的, 和环境无关, 能量变化在电子伏特级别上.

为便于分析问题, 在图2画出了 3 种基本的热力学 状态分布形状及它们在温度坐标上的移动(或漂移)现 象. 如果不改变蛋白质的稳定性绝对大小, 但改变蛋白 质对温度(或其他因素)的敏感性，则产生分布曲线的 漂移现象. 图2中横坐标可以是任何环境因素, 如温 


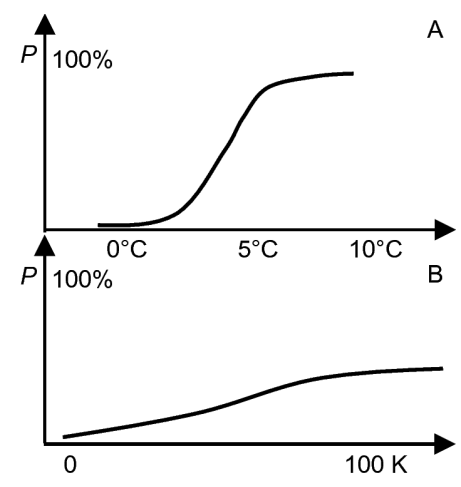

图 1 典型热力学体系状态的热力学分布与传统分子量子 能级的分布

$P$ 为电子在一能级或体系在某一状态的几率. $A$ : 热力学体系状态的 分布; B: 分子量子能级的电子分布

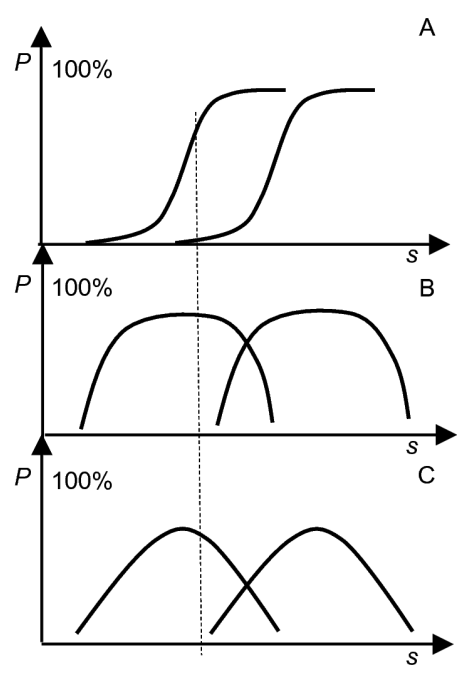

图 23 种基本的热力学状态分布及漂移现象

$P$ 为概率, $s$ 为环境刺激因子的强度. $\mathrm{A}:$ 体系 2 态分布的分布曲线; B: 体系多态分布中稳定能较大的状态的分布曲线，其在100\%处有平 台; C: 体系多态分布中稳定能较小的状态的分布曲线, 在 $100 \%$ 处没 有平台, 一般情况下这种状态的概率达不到 $100 \%$. 酶的活性构象的 分布曲线一般属于第 2 种，受体的活性构象一般属于第 3 种. 这与它 们的调节特性相一致

度、盐浓度、变性剂浓度和 $\mathrm{pH}$.

来源于不同生物种类的酶稳定性相差极大，来源 于高温菌的酶一般在高温才能发挥其活力，而来源于 低温菌的酶可以在低温下显示高活力 ${ }^{[17]}$. 本理论证明 这是酶活性构象在温度坐标上的漂移现象 ${ }^{[2]}$. 这种现 象由两种不同的原因引起: 第一为蛋白质稳定能的增 加, 第 2 为 $\Delta C \mathrm{p}$ 或 $m$ 值的减少 ${ }^{[18]}$. 与这种原理相关的现 象是一些高温酶(所有?)冷变性温度也较高 ${ }^{[5,19]}$.
如果连续改变蛋白质的稳定性, 其状态分布曲线 形状就会发生变化. 图3表示了二者之间的关系. 随着 体系状态的稳定能下降，分布曲线的100\%平台区开始 逐渐缩短, 然后这一区域消失, 体系的状态概率进一步 下降, 分布曲线成为钟状, 分布区域也逐渐缩小. 这一 关系已经得到理论与实验结果的完全证明 ${ }^{[20]}$.

体系状态的热力学振荡. 如果体系的两种状态热 力学稳定能量相近, 则体系的两种状态可以交替出现, 从而在两种状态中产生热力学振荡. 一个例子是离子 通道在开放与关闭状态中的转变 ${ }^{[21,22]}$. 体系状态的热 力学振荡不影响热力学平衡. 由于缺乏有效的观察手 段, 大量热力学振荡现象还没有被发现或研究. 传统 科学观点认为, 热力学特性是大量分子行为的统计学 特性, 从此现象可以得出结论, 热力学特性也可以是 一个分子行为在较长时间范围内的统计特性 ${ }^{[21]}$.

\section{2 蛋白质热力学体系之间的关系}

上面讨论了一个复杂热力学体系的原理与特点, 但是要用这些原理分析讨论蛋白质热力学运动的特点 与性质是不够的. 蛋白质热力学运动的特点是它包含 很多(理论上无穷多)的热力学体系, 因此有必要研究 其特点. 基于这种关系, 提出了蛋白质热力学结构理 论 $^{[3,5]}$. 蛋白质动态热力学结构理论的基本观点是: (i) 任何蛋白质分子不是一个均一的热力学体系, 而是由 众多(无限)热力学体系组成的复杂体系; (ii) 任何一 个热力学体系都有热力学独立性, 有形成独特三维结 构(蛋白质构象)的能力, 因此任一蛋白质分子有呈现 无限类型蛋白质构象的潜力; (iii) 不同热力学体系之 间的关系称为蛋白质热力学结构; (iv) 任一蛋白质的 热力学结构也是随环境不同而变化的. 由于蛋白质热 力学体系的关系是随环境的变化而变化的, 因此, 无 法给出具体的结论, 只能具体问题具体分析.

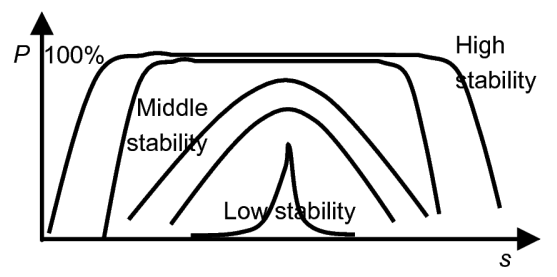

图 3 体系的稳定性与分布曲线的关系 $P$ 为概率, $s$ 为环境刺激因子的强度 
蛋白质内部两个不同的热力学体系可以完全独 立, 相互重叠或偶联. 如果这两个体系分属不同的层 次, 一个热力学体系可以包含另一个体系, 在功能上 表现生物调控机制. 蛋白质热力学体系的偶联现象与 酶的调节机制有关.

\section{3 酶催化反应动力学方程}

根据目前的知识, 酶催化反应的过程可以表示为

$$
\begin{aligned}
& (\mathrm{ES})^{\mathrm{IN}} \\
& \uparrow \downarrow \\
\mathrm{E}+\mathrm{S} \rightleftharpoons & \mathrm{ES} \rightleftharpoons(\mathrm{ES})^{*} \rightarrow \mathrm{EP} \rightarrow \mathrm{E}+\mathrm{P} \\
& \uparrow \downarrow \\
& (\mathrm{ES})^{\mathrm{IN}}
\end{aligned}
$$

其中 $\mathrm{E}$ 代表酶, $\mathrm{S}$ 为底物, $(\mathrm{ES})^{\mathrm{IN}}$ 为酶底物复合物非 活性构象(有很多种, 图中画出了两种), ES为活性构 象, (ES) ${ }^{*}$ 代表酶底物过渡态构象, P代表产物.

考虑到酶底物复合物代表一种热力学体系, 其能 量是变化的, 通过修改Arrhenius方程, 推导出酶反应 动力学方程

$$
\begin{aligned}
K & =\mathrm{APexp}\left\{-\left(\Delta G_{0}-b\left[T-T_{0}\right]\right) / k_{\mathrm{B}} T\right\} \\
& =\mathrm{AD}_{\mathrm{T}} \mathrm{P} \exp \left(-\Delta G_{a} / k_{\mathrm{B}} T\right),
\end{aligned}
$$

其中 $K$ 为酶反应速度常数, $k_{\mathrm{B}}$ 为Boltzmann常数, $\mathrm{A}$ 代表传统Arrhenius方程指前因子. $\Delta G_{0}$ 是在温度为参 考温度时的活化能. $\left(\Delta G_{0}-b\left[T-T_{0}\right]\right)$ 为温度 $T$ 时的活化 能, $\Delta G \mathrm{a}$ 为表观活化能. $\mathrm{AD}_{\mathrm{T}}$ 为酶学A Arrhenius方程表观 指前因子. $P$ 为酶底物活性构象的几率.

此方程与传统Arrhenius方程形式相同, 但其常数 物理学含义不同: 在本理论中, 酶反应的活化能不是 常数, 是一个变量, 而在传统Arrhenius方程中是常数. 酶学Arrhenius方程表观指前因子 $\left(\mathrm{AD}_{\mathrm{T}}\right)$ 也是变量, 随环 境变化而变化. 这样酶反应速率与酶分子构象热力学 参数就在此方程中建立了关系.

在实际中, 可以根据此方程推导出酶底物复合物 活性构象的分布曲线. 根据酶活性特性, 在图4中表出 了高温(嗜热生物中的酶)乙醇脱氢酶的各种性质 ${ }^{[2,19]}$.

酶在尿素溶液中的稳定性下降, 如果在任何情况 下其活性构象都达不到 $100 \%$. 其酶学动力学曲线将如 图5所示. 在这种情况下, Arrhenius plot得不到直线部

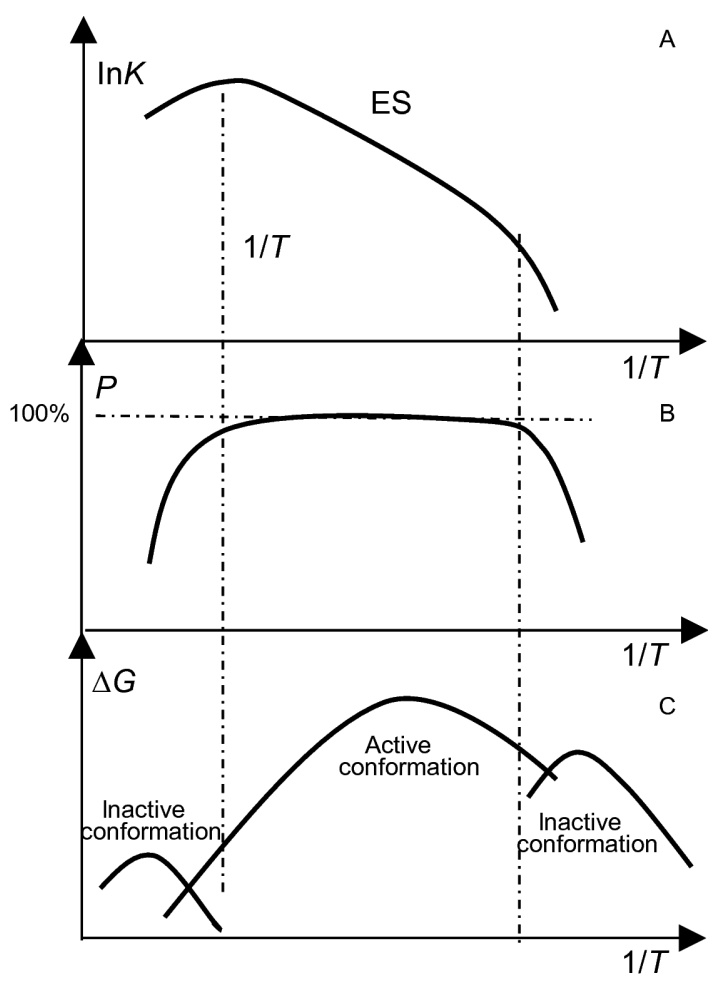

图 4 高温乙醇脱氢酶的各种性质

$P$ 为概率, $K$ 为酶学反应速度常数. A: 乙醇脱氢酶活性的Arrhenius plot; B: 酶底物复合物的活性构象的分布曲线; C: 酶底物复合物的 3种构象稳定性曲线. 此图根据酶的各种性质及蛋白质构象稳定性 方程绘制, 并没有实验数据. 此图与酶的各种性质完全吻合

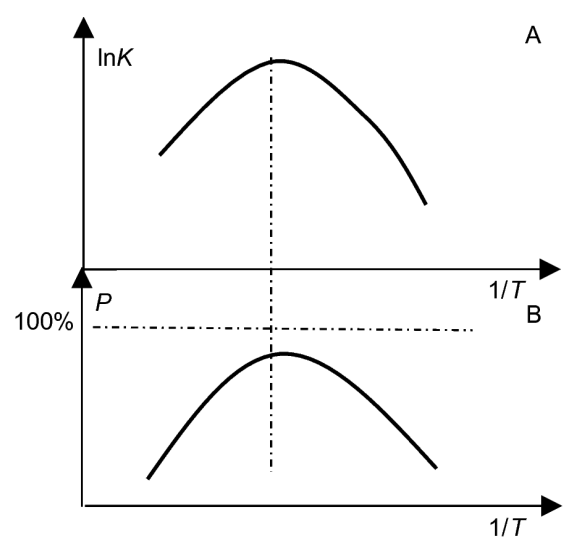

图 5 低稳定性酶的动力学特性

$P$ 为概率, $K$ 为酶学反应速度常数. A: 速度曲线图; B: 酶活性构象概 率分布图

分. 在整个温度范围内, 酶活性构象的几率是变化的. 在酶学历史上, 有酶动力学两相之说. 此假说认为 
有些酶在高温与低温下构象不同，所以在不同温度区 域的酶催化反应活化能不相同, 动力学特性不同 ${ }^{[19,23]}$. 本理论认为, 在低温下出现的新相是一种假象. 这是 由于酶在低温下变性引起的. 有关此相的任何热力学 数据都是没有任何科学意义的, 是一种错误解释 ${ }^{[19]}$. 邹承鲁曾观察到酶活力在低浓度尿素下升高的现象, 这种现象在很多酶中, 包括乙醇脱氢酶都观察到 ${ }^{[19,24]}$. 他认为这是尿素引起酶活性部位构象变化的结果. 本 研究组的观点是这种现象是由尿素引起酶活性构象概 率变化引起的. 这个观点与下列证据完全相符: ( i ) 这 种现象都是发生在低温，发生在酶活性构象与非活性 构象转变的温度, 如图5的低温段(Arrhenius作图非线 性区域); (ii) 在高温下(Arrhenius作图线性区域)观察 不到这种现象; (iii) 这种现象可有很多种物质诱导产 生; (iv) 尿素等蛋白质变性剂作用于蛋白质整体构象, 而非特异针对活性部位; (V) 很多酶的活性不受尿素 等变性剂的影响. 因此, 本研究组认为邹承鲁观察到的 所谓活性部位的柔性变化应当是酶非活性构象与活性 构象转变引起的, 是现象, 而非引起酶活性发生变化的 本质. 邹承鲁认为酶活性部位柔性为酶充分表现其催 化活性所必需, 本理论观点是蛋白质柔性(蛋白质热力 学状态或运动性)是调节酶活性构象几率的因素, 酶活 性构象只是在一定的蛋白质柔性下才能呈现.

\section{4 酶高效催化的热力学机制}

酶是一种特殊的生物化学催化剂, 能在常温下高 效催化化学反应, 其效率远高于一般的化学催化剂. 酶的催化原理是酶学的重要理论基础. Pauling ${ }^{[25]}$ 提出 酶与反应物过渡态结合能力强的观点, 因而酶能大幅 度降低反应的活化能. 这一思想被一些人称为酶学中 心法则. Fersht ${ }^{[26]}$ 在其著名酶学教科书中在实验科学 的基础上总结了酶高效催化的机制与原因，但没有在 热力学原理上加以阐明论证. 虽然得到很多人的推崇 并被写入教科书，Pauling的思想仅是一种想法，Pauling从酶催化反应的活化能减少这一事实出发考虑酶 的高催化活性问题是正确的，但他的假说无法定量说 明为什么活化能减少了, 而且酶与过渡态结合紧密结 论缺乏实验科学的判决性科学证据 ${ }^{[27]}$. 本研究组在体 系的热力学原理基础上，提出了酶降低活化能的新 理论.
按照本研究组的理论, 图6绘出了酶催化过程中不 同阶段酶底物复合物蛋白质构象的能量水平. 因此 $\Delta G_{\mathrm{a}}-\Delta G_{\mathrm{Ea}} \leq \Delta G_{\mathrm{B}}, \Delta G_{\mathrm{B}}$ 为酶与底物的结合能.

简单地说, 酶催化反应中活化能降低来源于酶与 底物的结合能力. 酶与反应物过渡态的结合能力低于 酶与底物的结合能力. 因此Pauling的假说是完全没有 必要的. 虽然酶与底物的结合降低了溶液总体系的自 由能, 但在化学反应方向能量却升高了, 这样就减少 了反应的活化能.

本研究组的观点与实验科学观点完全一致. 酶与 底物复合物, 虽然降低了酶溶液的总自由能, 但在化 学反应度量下, 可以引起底物形变, 导致化学键应力 紧张; 另一方面使化学反应的基团相互靠近 ${ }^{[26]}$. 这些 有利于化学反应的因素都是以酶与底物的结合能力为 代价得到的.

从理论上可以这样考虑, 酶底物复合物的构象构 成一个集合 $\left(\mathrm{S}_{\mathrm{ES}}\right)$, 酶与反应物过渡态的结合构象亦构 成一个集合 $\left(\mathrm{S}_{\mathrm{ESTS}}\right), \mathrm{S}_{\mathrm{ESTS}}$ 是 $\mathrm{S}_{\mathrm{ES}}$ 一部分. 显然, $\mathrm{S}_{\mathrm{ESTS}}$ 的构 象处于高能态. 这个原理结合酶可以同时利用不同化 学机制的现象, 如溶菌酶可以同时利用酸碱催化机制, 就可以对酶的高催化能力做出完善的热力学说明 ${ }^{[29]}$.

在酶反应模型中，酶催化反应的过渡态是酶底物 复合体的过渡态, 而在Pauling中是底物的过渡态. 在 催化过程中, 酶分子构象需要做出一系列的改变, 这 是Pauling在当时所不能认识的.

\section{5 蛋白质运动与酶活性的间接逻辑学关系}

在实验生物科学领域, 结构生物学占统治地位, 由 于理论与技术的缺乏, 对这个命题科学意义的疑问等 多重原因, 研究蛋白质运动与功能的关系没有得到重 视. 但在理论科学领域, 理论物理学家坚信生物学原 理需要用热力学原理来解释, 因此研究蛋白质热力学 运动与生物功能的关系成为科学的重点方向 ${ }^{[30-32]}$. 因 此对这个科学命题的研究有关物理学理论及生物学理 论发展的方向, 所以有关争论绝不是空穴来风, 毫无意 义, 相反是意义重大的, 是孕育新科学理论的诞生. 美 国政府对这个领域的科学家支持最大, 美国科学家介 入的人最多, 因此火爆的科学争论就发生在美国. 因 为这牵涉对热力学理论的理解, 所以争论的都是出身 物理、化学的科学家. 


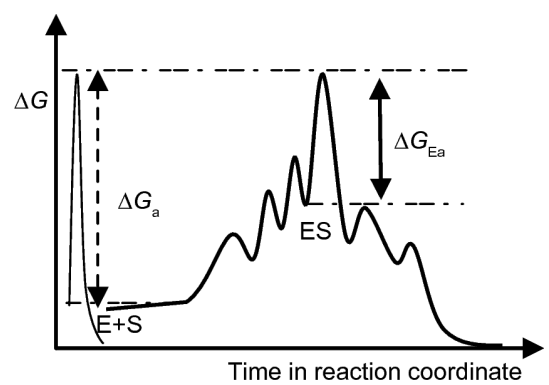

图 6 酶催化过程中的能量图标(参考文献[28], 并做修改)

左侧是没有催化的反应能级图, 右侧为酶催化的能级图. $\Delta G_{\mathrm{a}}$ 代表没 有催化的化学反应活化能, $\Delta G_{\mathrm{Ea}}$ 为酶催化反应的活化能

从科学原则上考虑, 我国科学家钱学森思想亦有 关这个科学命题. 钱学森以敏锐的科学目光, 深刻的 科学思想, 深深感觉到用热力学原理解释生物学原则 的困难与局限，因此不断提出新思想、方法，并试图 解决这个问题. 根据笔者的了解, 钱学森没有从修改 基础热力学原理出发，而是沿着Prigogine的道路，试 图利用复杂性科学问题解决这个问题. 虽然这种科学 道路很难说是完全错误的，但至少是不成功的，富有 争论的. 但在理论物理领域, 这代表主流看法. 目前这 种科学道路与生物学实验科学领域还没有建立真正的 科学联系. 在这个理论方向中, dynamic system的过程 或性质是通过数学模拟解决的, 因而存在体系内部运 动与体系结构之间的直接逻辑关系.

在蛋白质、酶学领域, 对有关问题, 即蛋白质运动 与酶活性的关系问题的探讨形成了 3 种不同的道路与 结论.

第1种以美国本土科学家SJ Benkovic (Pennsylvania State University), JP klinman (Berkeley)为代表, 他 们试图通过改进实验仪器, 利用不同实验科学方法, 寻 找更多的科学证据支持蛋白质运动与酶活性之间的关

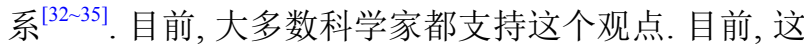
个学派发现了有关此科学命题的大量实验事实，但是 这个学派在围绕这个科学命题成立的科学范型, 科学 理论等方面基本没有涉及. 因此他们对于Warshel的批 判与责难完全没有反驳的能力.

第2种以归化美国人Arieh Warshel (University of Southern California)为代表. Warshel在理论上断然否定 了蛋白质运动与酶活性之间的逻辑学联系 ${ }^{[36,37]}$. 他的 基本论点非常简单: 如果蛋白质运动和酶的活性相关,
那么酶催化反应的活化能及酶动力学参数就会随蛋白 质运动的不同而发生改变. 而所有的证据并不支持酶 活性与蛋白质运动直接相关的观点, 实验观察到酶活 性与蛋白质运动相关的报道都可以解释为表面现象, 而非必然性逻辑学联系. 他争论的缺陷是他不能给反 面证据提出合理的解释，另外，他人为陷入为争论而 争论的境地, 有人持这种看法: “他不是为了解决科学 问题, 而是把证伪同行的工作作为自己科学研究的指 南”, 因此这些争论超出了科学道德许可的限度.

第3种为本研究组的科学道路. 在1986, 吉林大学 酶工程国家重点实验室程玉华教授就告诫笔者“在理 论上有可能将众多的酶学模型与蛋白质科学概念统一 起来，这种统一必然是建立在蛋白质柔性或运动性基 础上的”, 同时鼓励笔者将此作为科研的长期方向. 经 过与程玉华教授讨论, 当时形成了这么一个看法: 科学 概念及其相互之间的逻辑学关系是理论科学研究的范 畴, 具体的实验科学结果办不到这一点, 因此需要建立 一种新的科学范型, 并在此理论范型中证明或否决这 种关系. 这一认识直接决定了笔者以后科学道路及思 想发展过程. 在此认识基础上, 通过对基础热力学原 理的修改, 得到了蛋白质体系热力学原理及修改后的 酶动力学方程, 在这个方程中, 蛋白质运动与酶活性 的关系得到逻辑学的确认并能解释大量实验现象. 但 是，本理论证明存在蛋白质运动与酶活性之间的间接 逻辑学关系, 而非直接性逻辑学关系, 具体说, 蛋白质 运动的改变影响蛋白质各种热力学体系的热力学特 性, 影响蛋白质热力学状态的分布, 从而间接影响酶的 活力. 在一些情况下, 如果蛋白质运动的改变没有影响 蛋白质热力学体系状态, 那么就会出现一些蛋白质运 动与酶活性无关的现象. Arieh Warshel的疑问在本理 论中自然得到解决, 因为可以通过活化能的改变, 酶动 力学中 $K_{\mathrm{m}}$, 酶学Arrhenius方程表观指前因子变化证明 这种关系.

为了更好地分析问题，把一些酶学、蛋白质热力 学科学概念及相互关系标识在图7中. 这些关系已经 得到理论与实验科学的多方面证明 ${ }^{[2]}$. 在这些关系中, 由蛋白质柔性引起的 $K_{\mathrm{m}}$ 改变在本质上等同于蛋白质配体的相互作用. 蛋白质运动性(柔性)可以改变 $K_{\mathrm{m}}$, 在 酶学实验中, 已知尿素浓度, 温度变化可以改变酶的 $K_{\mathrm{m}}$. 其他关系在本文其他地方介绍. 


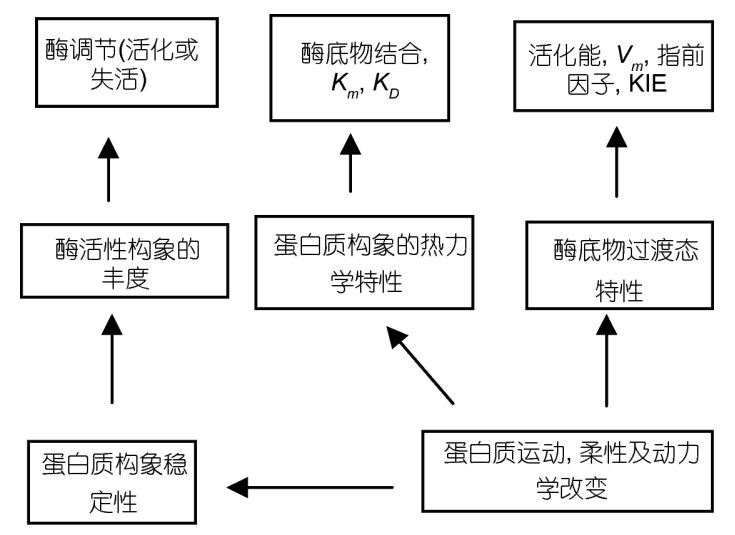

图 7 蛋白质酶学各个概念的逻辑学联系

\section{6 酶活性相关性运动位点}

在酶分子中, 不是所有的蛋白质运动都和酶的活 性息息相关. 有些是完全无关的. 其中一些与酶活性 相关的运动位点被命名为dynamic $\operatorname{sit}^{\left[{ }^{[7]}\right.}$. 图8显示了酶 dynamic sites和活性部位的关系.

酶蛋白质运动部位(dynamic site)或易动部位一般 远离酶的活性部位. 根据蛋白质动态热力学理论, 一个 酶分子有 $5 \sim 10$ 个的易动部位. 2 个酶的深入研究得到和 这个结论完全一致的结果 ${ }^{[38,39]}$. 通过调节部位的运动 性，可以调节酶的活性，称酶的这种调节机制为别动 调节(allodynamic regulation). 酶的调节物可以通过结 合到酶的易动部位, 影响其运动性, 酶学过渡态热力学 性质, 从而影响酶的活性.

可以这样理解酶的易动部位的功能. 酶从和底物 碰撞开始，通过酶底物复合物的各种不同蛋白质构象， 酶底物的过渡态, 酶与产物复合物, 又回到裸体酶, 之 间经历很多步蛋白质构象变化. 如果把酶分子在催化 反应经历的构象轨迹称为轨道的话，那么任何影响这 个轨道的因素都可以影响酶的活性. 有些部位对蛋白 质构象的影响很大，因而就可显著影响酶构象变化的 轨道, 这些部位就是酶的易动部位. 从理论上考虑, 酶 的易动部位对应于一些flexible loop, 无规则卷曲部位 (disorder area). 这些部位的运动性可改变酶分子蛋白 质构象集的大小、范围，进而影响蛋白质构象变化的 轨道, 蛋白质热力学结构的重构造过程.

别动调节与别构调节的区别是别动调节是通过调 节蛋白质运动性实现的. 别动调节不需要蛋白质几何

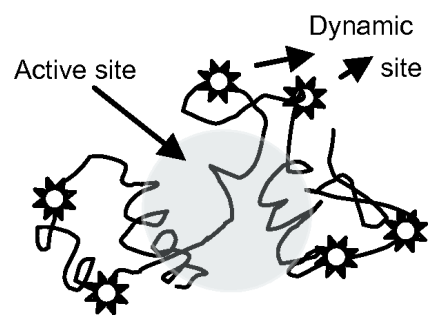

图 8 酶的运动部位及活性部位

学结构的改变, 而这是别构调节绝对必需的. 当然蛋 白质几何学结构的改变并不与别动调节理论相矛盾. 在理论上，别动调节是酶分子蛋白质热力学结构重构 造的结果. 而别构调节是酶分子蛋白质几何学结构重 构造的结果. 别动调节机制的一个优点是它能处理多 个位点对酶活性的影响及其他们之间的相互作用 ${ }^{[0-11]}$.

\section{7 蛋白质运动与蛋白质稳定性的关系}

蛋白质、酶的稳定性与运动的关系也是蛋白质科 学的重大问题. 蛋白质的稳定性分为热力学稳定性与 动力学稳定性. 在此讨论的是热力学稳定性. 传统观 点是存在蛋白质运动与稳定性的直接性逻辑学关系, 也就是蛋白质运动加剧就会导致蛋白质、酶失去热力 学稳定性, 从而导致蛋白质变性. 这个观点与大量实验 科学结果相吻合, 并得到大量科学家的支持. 但是也有 一些少量的实验结果并不支持这一观点，因此在此问 题上陷入了争论 ${ }^{[00,41]}$, 问题长期得不到解决.

本理论证明，存在蛋白质运动性与稳定性的间接 性关系．这种观点能解释目前所有的科学证据，而又 没有发现于此相反的科学证据. 这种关系可在图9中 得到阐明。

在生物化学上，一般蛋白质稳定性指蛋白质几何 构象的稳定性, 而非热力学稳定性，在实验上以圆二 色性(circular dichroism, CD)作为指标. 因此它们都可 以用图9A来代表. 因为图9B和 $C$ 给出同样的统计学结 果, 因此蛋白质几何构象稳定性与热力学运动没有直 接关系. 也可这样分析，蛋白质几何构象的稳定性决 定于蛋白质运动在不同蛋白质构象之间的相对强度, 而非其绝对强度. 


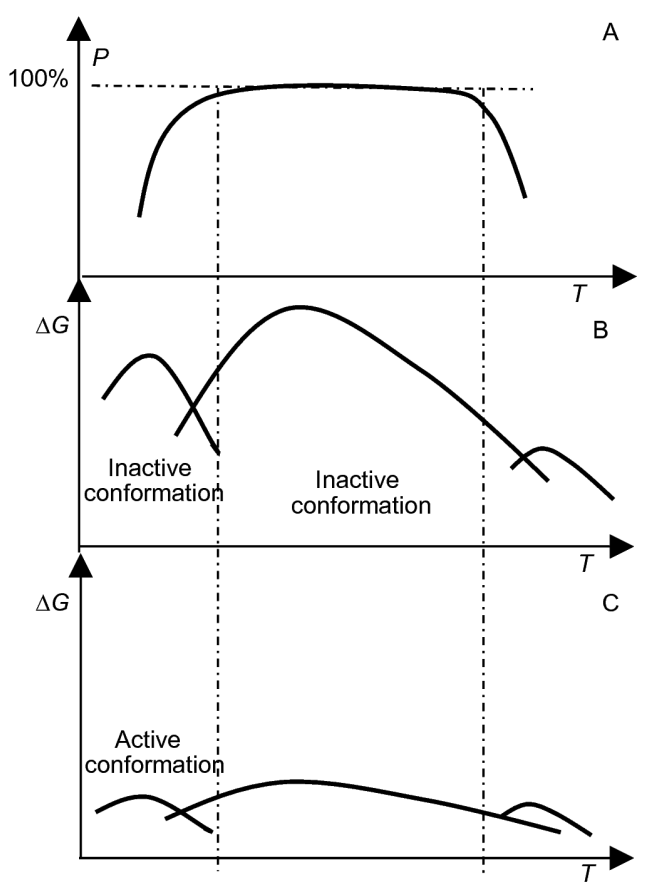

图 9 酶分子活性构象分布曲线与蛋白质稳定性曲线

$\mathrm{P}$ 为概率. $\mathrm{A}$ : 酶活性构象在温度坐标下的分布曲线; B和 C: 两种不同 蛋白质构象热力学稳定性曲线, 两种蛋白质热力学稳定性差别极大, 运动特性差别很大, 但 $\mathrm{B}$ 和 $\mathrm{C}$ 的热力学统计结果都会得到 $\mathrm{A}$. 此图根 据大量酶热力学数据及蛋白质稳定性曲线绘制 ${ }^{[18,42]}$

\section{8 酶的热力学调节机制}

上面在蛋白质运动性基础上讨论了酶调节的原 理一一别动调节(allodynamic regulation). 但是完整的 酶调节理论需要在热力学基础上讨论，或在酶分子活 性构象分布基础上考虑 ${ }^{[9 \sim 11]}$. 因此进一步提出了酶蛋 白质调节的热力学原理或模型. 其基本原理为任何物 质，如活化剂、抑制剂，或物理学因素，如 $\mathrm{pH}$ 、盐离 子、尿素, 通过影响酶蛋白质活性构象的热力学性质 和热力学分布而影响或调节酶的活性. 其具体变化机 理可以通过测定，分析酶分子活性构象的分布曲线 获得.

从理论上考虑, 有两种基本分子生物学机制: 酶的 特异性抑制剂或激活剂通过结合到蛋白质某一特定部 位, 从而改变蛋白质运动特性及活性构象的概率. 另一 种是通过非特异性相互作用，改变蛋白质结合水的分 子簇或蛋白质链的结构从而改变酶蛋白质构象，这一 类包括温度，全身性吸入麻醉药，低分子量有机物质， 尿素或蛋白质变性剂, 盐离子. 酸碱度或盐离子能通过
两种作用方式作用, 在此情况下, 需要具体实验结果才 能判定.

一般而言, 有很多种因素可以调节酶的活性. 这些 因素可以同时作用在同一酶分子上，其作用或发生整 合. 一些会有协同作用，一些会有相互抑制作用. 这些 问题需要具体问题具体分析, 目前没有发现规则. 对通 过非特异性相互作用调节酶活性的因素而言, 它们对 酶分子，蛋白质受体活性的影响或调节作用是可以叠 加的. 这是挥发性麻醉药的特性.

蛋白质, 酶基因改变, 磷酸化等修饰也是蛋白质、 酶的调节方式. 它们的作用原理可以用图10表示. 图中 $M$ 代表突变酶的活性构象分布曲线, $N$ 代表天然或野生 型受体的活性分布曲线. 由于基因突变或修饰改变了 蛋白质热力学性质，使受体或酶的活性构象在某一物 理学坐标 $(s)$ 下发生了漂移, 这样, 在给定的生理学条 件或活力测定条件下，两种蛋白质酶、受体分子的活 力发生了巨大变化. 在某些情况下, 或发生无活性到 完全活性的转变(本图仅描述基因突变引起的别动调 节机制变化原理，并非所有的蛋白质基因突变都可用 这个原理描述, 在图中 $M$ 与 $N$ 可以有差别).

考虑到蛋白质不是一个热力学体系, 而是包含多 个热力学体系, 在图11画出几个代表性的体系及相互 关系. 一个蛋白质含有很多调节部位, 通过结合调节 物或相应刺激因素调节构象可发生变化, 这些变化能 影响活性构象的热力学几率，从而影响蛋白质或酶的 活性. 调节构象为局部构象, 活性构象为高级构象形 式. 活性构象与非活性构象的转变可由体系的配分函 数来描述, 但调节构象与活性构象的关系必须由蛋白 质动态热力学结构理论来分析. 不同的调节构象的作 用可以相互影响. 在本理论中, 受体激动剂属于正向 调节剂, 仅它是天然配体罢了, 在热力学中没有任何 特殊性.

另一个重大的问题是蛋白质、酶、受体的活力构 象曲线与其生物功能要求完全一致. 在图12标出了草 鱼(Ctenopharyngodon idellus) GABA受体和NMDA受 体的活性构象分布曲线. 在体内, NMDA受体是主要的 兴奋性受体, GABA是主要的抑制性受体. 当温度升高 时, 生物体对兴奋性受体的活性要求降低, 对抑制性受 体的活性要求则升高, 所以NMDA活性下降, 而GABA 受体的活力则升高. 这样这两种受体的热力学行为与 生物体的代谢调节方式完全吻合 ${ }^{[5,8]}$. 数据提示, 在高 


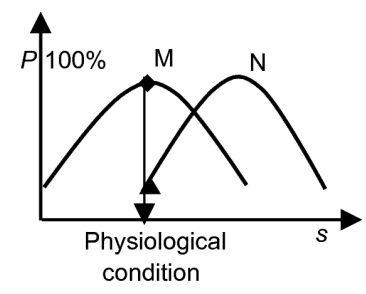

图 10 基因突变影响受体、酶调节作用的原理 $P$ 为概率, $s$ 为一环境因子的强度

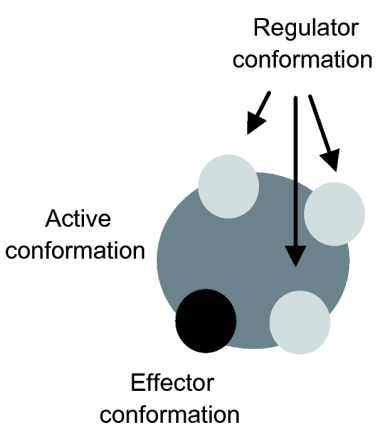

图 11 理想受体(或酶)蛋白质动态热力学结构

酶学中effector conformation指酶的活性部位. 这个图有很多变化形 式, 但在本文不讨论, 请参考文献[12]

温下人陷入昏迷是由NMDA受体完全失活引起的 ${ }^{[8]}$.

\section{9 生物信息的热力学整合机制}

上面谈到, 在蛋白质与不同配体相互中, 在蛋白质 响应环境因子变化中，会出现不同生物信息(作用)在 蛋白质分子中的整合现象. 其基础热力学原理非常简 单，不同的作用转化为热力学信息，在此基础上进行 热力学整合. 但由于热力学整合受到蛋白质结构的影 响，所以不同的蛋白质显示不同的性质与特征. 热力 学整合机制两个极端典型的现象是协同与抑制. 这里 不讨论生物信息整合具体现象与机制，仅就生物信息 流一般现象原理做出解释.

从蛋白质角度考虑，基因是最基础的生物信息源. 基因包含了蛋白质一级序列信息、结构信息、折叠信 息及对环境的依赖关系或蛋白质热力学稳定性信息 一旦基因序列翻译成蛋白质多肽链，基因生物学信息 转变成蛋白质序列信息, 就开始了生物信息的热力学 整合过程. 在此过程中，蛋白质序列信息转变成蛋白 质残基的热力学信息，蛋白质各个氨基酸残基的热力 学信息经过局部(local)区域和整体区域的多层次整合,
最后形成蛋白质各种热力学体系及蛋白质几何学结 构. 各种不同的蛋白质种类和其他物质经过多层次整 合, 最后形成各种生物学组织或器官.

图13标出了酶蛋白分子在各个层次上的热力学性 质, 从图中可以看出, 由于热力学整合作用, 不同层次 的热力学体系的特性完全不同.

热力学信息的整合机制与分子生物学中心法则是 不兼容的. 本研究组认为分子生物学法则在生物信息 整合领域完全不成立. 分子生物学中心法则仅适用于 核酸的复制、转录、蛋白质翻译过程 ${ }^{[44]}$. 另外，分子 生物学中心法则与体系生物学基本原理无法兼容，这 也是其重大理论缺陷 ${ }^{[12,45 \sim 47]}$

根据不可逆热力学及自然界秩序起源的理论，在

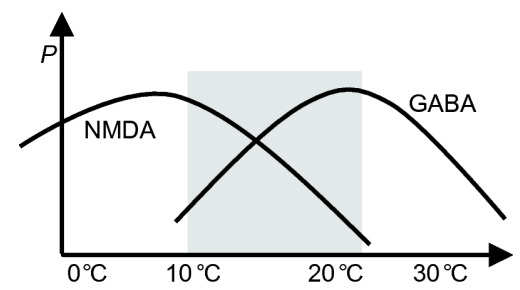

图 12 NMDA和GABA受体的活性构象的分布 $P$ 为概率. 阴影部分为鱼的生理学范围

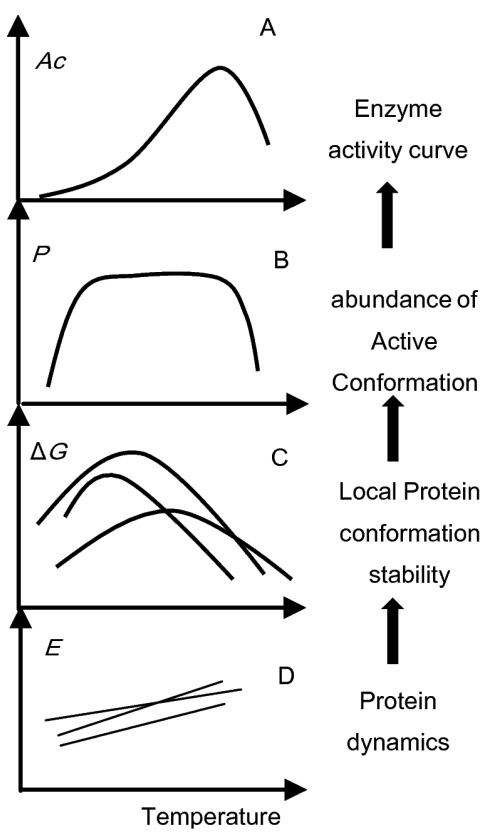

图 13 生物信息在不同层次的体现

$A c$ 为酶活性, $E$ 为能量. $\mathrm{A}$ : 酶活性曲线; $\mathrm{B}:$ 酶活性构象分布曲线; $\mathrm{C}$ : 蛋白质局部构象稳定性曲线 ${ }^{[43]} ; \mathrm{D}$ : 氨基酸残基的热力学运动幅度 
生物学层次结构中低层次生物信息量最大, 在高层次 上较少(以能量变化计算)。很多低层次的生物信息在 高层次中没有任何意义 ${ }^{[12]}$. 在理论上可推出一个结论, 在相同的基因背景下，可以产生很多种蛋白质构象或 功能，但具体到某一情况下，或可仅是一种或少数几 种. 但这需要生物信息与环境因素的信息相互之间的 整合作用(基因与环境互作).

综上所述, 复杂热力学体系及蛋白质热力学概念 能完全给予酶学, 蛋白质不同性质以合理的解释, 并
把不同的科学概念与性质在逻辑学上联系起来. 在理 论上, 复杂热力学体系完全适用于生物信号网络体 系、生理学体系、生态学体系等, 但这远超出此文的 范围. 过去, 研究蛋白质热力学的人很少, 蛋白质热力 学没有和蛋白质、酶功能联系起来. 随着科学的发展 及这些问题的解决, 蛋白质热力学已经成为生物物理 学、生物化学、蛋白质科学及理论生物学的基础理 论. 以后任何生物学现象与功能需要在分子层面上做 出热力学的解释.

\section{参考文献}

1 Karplus M. Aspects of protein reaction dynamics: deviations from simple behavior. J Phys Chem B, 2000, 104: 11-27

2 Zhao Q. On the indirect relationship between protein dynamics and enzyme activity. Prog Biophys Mol Biol, 2017, 125: 52-60

3 Zhao Q. Protein thermodynamic structure. IUBMB Life, 2009, 61: 600-606

4 Zhao Q. Partition function of the protein conformational state. Jnl Comp Theo Nano, 2012, 9: 745-751

5 Zhao Q. Nature of protein dynamics and thermodynamics. Rev Theor Sci, 2013, 1: 83-101

6 Zhao Q. Protein flexibility as biosignal. Crit Rev Eukaryot Gene Expr, 2010, 20: 71-84

7 Zhao Q. dynamic model of enzymes action. Protein Pept Lett, 2011, 18: 92-99

8 Wang A, Zhang Z, Zhao Q. In vivo measurement of protein functional changes. Int J Biol Sci, 2009, 5: 411-420

9 Zhao Q. A thermodynamic and theoretical view for enzyme regulation. Biochemistry (Moscow), 2015, 80, 1-7

10 Zhao Q. Allodynamic regulation of protein activity. Quant Matt, 2013, 2: 144-152

11 Zhao Q. Classification of enzyme regulators within thermodynamic model of enzyme regulation. Mol Enz Drug Tar, 2016, 2: 14

12 Zhao Q. Nonalgorithmicity and algorithmicity of protein science. Adv Biosci Biotech, 2011, 02: 340-346

13 Zhao Q. Irreversible thermodynamics theory of protein folding and protein thermodynamics structure. Prog Biochem Biphys, 2001, 28: 429-435

14 Frauenfelder H, Sligar S G, Wolynes P G. The energy landscapes and motions of proteins. Science, 1991, 254: 1598-1603

Elber R, Karplus M. Multiple conformational states of proteins: a molecular dynamics analysis of myoglobin. Science, 1987, 235: 318-321

Maity H, Maity M, Krishna M M G, et al. Protein folding: the stepwise assembly of foldon units. Proc Natl Acad Sci USA, 2005, 102: 4741-4746

Razvi A, Scholtz J M. Lessons in stability from thermophilic proteins. Protein Sci, 2006, 15: 1569-1578

Myers J K, Nick Pace C, Martin Scholtz J. Denaturant $m$ values and heat capacity changes: relation to changes in accessible surface areas of protein unfolding. Protein Sci, 1995, 4: 2138-2148

19 Nagel Z D, Dong M, Bahnson B J, et al. Impaired protein conformational landscapes as revealed in anomalous Arrhenius prefactors. Proc Natl Acad Sci USA, 2011, 108: 10520-10525

20 Pace C N, Tanford C. Thermodynamics of the unfolding of $\beta$-lactoglobulin A in aqueous urea solutions between 5 and 55. Biochemistry, 1968, 7: 198-208

21 Zhao Q. A thermodynamic model of protein signaling network. Rev Theor Sci, 2016, 4: 1-9

22 Lape R, Colquhoun D, Sivilotti L G. On the nature of partial agonism in the nicotinic receptor superfamily. Nature, 2008, 454: 722-727

23 Gregolin C, Ryder E, Lane M D. Liver acetyl coenzyme A carboxylase I. isolation and catalytic properties. J Biol Chem, 1968, 243: 4227-4235

Tsou C L. Conformational flexibility of enzyme active sites. Science, 1993, 262: 380-381

Pauling L. Chemical achievement and hope for the future. Am Sci, 1948, 36: 51-58

Fersht A. Structure and Mechanism in Protein Science: a Guide to Enzyme Catalysis and Protein Folding. London: Macmillan, 1999 
Benkovic S J, Hammes-Schiffer S. A perspective on enzyme catalysis. Science, 2003, 301: 1196-1202

Zhao Q. The Origin of Natural Order: An Axiomatic Theory of Biology. Singapore: World Scientific Press, 2017

钱学森, 于景元, 戴汝为. 一个科学新领域——开放的复杂巨系统及其方法论. 北京: 中国系统工程学会第六次年会, 1990. 526-532

Klinman J P, Kohen A. Hydrogen tunneling links protein dynamics to enzyme catalysis. Annu Rev Biochem, 2013, 82: 471-496

Henzler-Wildman K A, Thai V, Lei M, et al. Intrinsic motions along an enzymatic reaction trajectory. Nature, 2007, 450: 838-844

Kamerlin S C L, Warshel A. At the dawn of the 21st century: is dynamics the missing link for understanding enzyme catalysis? Proteins, 2010, 78: $1339-1375$

Schrank T P, Bolen D W, Hilser V J. Rational modulation of conformational fluctuations in adenylate kinase reveals a local unfolding mechanism for allostery and functional adaptation in proteins. Proc Natl Acad Sci USA, 2009, 106: 16984-16989

39 Henzler-Wildman K A, Lei M, Thai V, et al. A hierarchy of timescales in protein dynamics is linked to enzyme catalysis. Nature, 2007, 450: 913916

40 Hernandez G, Jenney F E, Adams M W W, et al. Millisecond time scale conformational flexibility in a hyperthermophile protein at ambient temperature. Proc Natl Acad Sci USA, 2000, 97: 3166-3170

41 Jaenicke R. Do ultrastable proteins from hyperthermophiles have high or low conformational rigidity? Proc Natl Acad Sci USA, 2000, 97: 29622964

42 Becktel W J, Schellman J A. Protein stability curves. Biopolymers, 1987, 26: 1859-1877

Huyghues-Despointes B M, Pace C N, Englander S W, et al. Measuring the conformational stability of a protein by hydrogen exchange. Protein Structure Stability Folding, 2001, 168: 69-92

44 Crick F. Central dogma of molecular biology. Nature, 1970, 227: 561-563

45 Mattick J S. Challenging the dogma: the hidden layer of non-protein-coding RNAs in complex organisms. Bioessays, 2003, 25: 930-939

46 Powell A, Dupré J. From molecules to systems: the importance of looking both ways. Stud Hist Philos Biol Biomed Sci, 2009, 40: 54-64

47 Shneider A M. Four stages of a scientific discipline four types of scientist. TIBS, 2009, 34: 217-223 


\title{
Physical characteristics of complex thermal system and working mechanism of enzymes
}

\author{
ZHAO QinYi \\ Medical Institute, CRRC, Beijing 100068, China
}

This paper discusses the behaviors and thermodynamic principle of complex thermal systems. The equations of enzymatic kinetics are revised to incorporate the thermodynamic parameters of protein conformational state, so that the role of protein conformational change in enzymatic reaction can be analyzed thermodynamically. In addition, the genetic interactions during the informational flow from genome to biological functions are analyzed thermodynamically. Based on these, the working mechanisms of enzyme action and regulation are further interpreted from a thermodynamic point of view and are found to be fundamentally the same. For example, the decrease of activation energy in enzymatic reaction comes from the binding between substrate and enzyme. Finally, we conclude that protein thermodynamics is a theoretical foundation for all biological processes.

protein, enzyme, regulation, mechanism, system

doi: $10.1360 / \mathrm{N} 052017-00298$ 